\title{
Approvisionnement En Eau De Consommation Et Récurrence Des Maladies Hydriques Dans La Commune De Bangangté (Ouest-Cameroun)
}

\author{
Nya Esther Laurentine, \\ Doctorante, Département de Géographie, \\ Université de Yaoundé I, Cameroun \\ Mougoué Benô̂t, \\ HDR, Maître de Conférences, Département de Géographie, \\ Université de Yaoundé I, Cameroun
}

Doi:10.19044/esj.2020.v16n11p115 URL:http://dx.doi.org/10.19044/esj.2020.v16n11p115

\section{Résumé}

Approvisionner les ménages camerounais des grandes agglomérations et des villes moyennes en eau potable est une préoccupation constante des pouvoirs publics. Malgré l'implication des acteurs dans l'amélioration de l'accès à l'eau potable, le problème d'eau dans la Commune de Bangangté est très épineux et constitue un risque sanitaire. Le but de cette recherche est de montrer le lien qui existe entre la qualité de l'eau consommée et la récurrence des maladies hydriques dans cette commune. Les enquêtes ont été effectuées auprès de 339 chefs de ménage tirés de manière aléatoire dans 4 villages de la commune : Bangangté, Bangoua, Bamena, Batchingou. Les informations sur les maladies hydriques recueillies par l'enquête-ménage ont été complétées par les données compilées des registres de consultation des différentes formations sanitaires et les analyses physico-chimiques et bactériologiques de 12 échantillons d'eau prélevés. Il ressort de cette étude que les eaux de sources et de bornes-fontaines les plus fréquemment consommées sont contaminées par les Escherichia coli, les streptocoques fécaux et les salmonelles qui rendent les populations vulnérables aux maladies telles que la typhoïde (57\%), l'amibiase $(47,2 \%)$ et les diarrhées $(45,5 \%)$. La réduction de ces maladies nécessite une gestion préventive des risques à la source de captage et une éducation des populations sur les méthodes de potabilisation de l'eau.

Mots clés: Approvisionnement, eau de consommation, vulnérabilité, maladies hydriques, Bangangté 


\title{
Drinking Water Supply and the Recurrence of Water-Borne Diseases in the Bangangte Municipality (West Region-Cameroon)
}

\author{
Nya Esther Laurentine, \\ Doctorante, Département de Géographie, \\ Université de Yaoundé I, Cameroun \\ Mougoué Benoît, \\ HDR, Maître de Conférences, Département de Géographie, \\ Université de Yaoundé I, Cameroun
}

\begin{abstract}
Providing Cameroonian households in big cities and medium-sized cities with drinking water is a constant concern of the public authorities. Despite the involvement of stakeholders in improving access to drinking water, the water problem in the Bangangté municipality is very difficult and constitutes a health risk. This study aims to show the link between the quality of water consumed and the recurrence of water diseases in this municipality. The surveys were carried out with 339 heads of household drawn at random in 4 villages of the municipality: Bangangté, Bangoua, Bamena, Batchingou. The information on water-borne diseases collected by the household survey was supplemented by the data collected in the consultation register of the various health facilities and the physico-chemical and bacteriological analyzes of 12 taken water samples. This study shows that the most frequently consumed springs water and standpipes are contaminated with Escherichia coli, faecal streptococci and salmonella, which makes populations vulnerable to diseases such as typhoid (57\%), amoebiasis (47.2\%) and diarrhea (45.5\%). The reduction of these diseases requires preventive management of the risk at the source of catchment and an education of the populations on the methods of water purification.
\end{abstract}

Keywords: Supply, drinking water, vulnerability, water-borne diseases, Bangangté

\section{Introduction}

L'eau est une ressource vitale essentielle aux êtres humains et à la nature. En 2015, 844 millions de personnes dans le monde n'ont pas accès à des services élémentaires d'approvisionnement en eau de boisson 
(UNICEF/OMS, 2017). La difficulté pour les ménages habitant les quartiers périphériques à se connecter au réseau d'eau potable pour des raisons de coût de branchement élevé (Mougoué, 1982) les oblige à recourir aux eaux de puits, de sources, ou de marigot (Nya, 2014). Adeline et Amirault (1998) soulignent que le recours aux eaux de sources et puits tient à plusieurs facteurs parmi lesquels l'insuffisance du réseau dans certains quartiers et le doute sur la qualité de l'eau distribuée par le concessionnaire. L'eau des sources et des puits apparaît comme étant la plus fiable aux yeux de la population qui la préfère à l'eau de robinet à cause de sa limpidité (Nnanga et al, 2014). Dans les villes, la plupart des ménages ont une préférence pour l'eau des puits et des sources parce que bien que la qualité ne soit pas garantie, ces points d'eau sont plus accessibles tant sur le plan financier que technique (Djeuda et al, 2001).

De la consommation d'une eau polluée, découlent les maladies d'origine hydrique. OMS/UNICEF (2000) et Beninguissé (1993) reconnaissent que l'insuffisance de la distribution moderne d'eau potable dont le corollaire est la persistance des modes traditionnels d'approvisionnement en eau dans les villes est lourde de conséquences sur la santé humaine. Pour corroborer cette affirmation, l'UNICEF (2006) affirme que la diarrhée constitue la deuxième cause de mortalité infanto-juvénile. Chaque année, elle tue environ 1,9 million d'enfants âgés de moins de 5 ans, principalement dans les pays en développement.

Au Cameroun, les dépenses liées aux maladies hydriques par ménage sont évaluées à 65975 F CFA (GWP-MINEE, 2009c). Dans la ville de Bangangté et sa périphérie, les chefs de ménage dépensent en moyenne 10000 F CFA par mois pour le traitement des maladies liées à l'eau de consommation (Nya, 2014). La consommation des eaux de qualité inacceptable expose les populations à de graves problèmes de santé publique. À partir d'une analyse des modes d'approvisionnement en eau dans la Commune de Bangangté et les raisons du choix d'un point d'eau, cette étude vise à montrer l'impact de la consommation d'une eau de qualité douteuse sur la santé.

\section{Méthodes et matériels}

\section{I.1. Méthodes}

La méthode hypothético-déductive et l'approche écosystémique de la santé humaine (ECOSANTE) ont été adoptées pour l'élaboration de ce travail. Ces démarches scientifiques s'appuient sur la collecte des données de sources secondaires et primaires.

\section{I.1.1. Recherche documentaire}

La collecte des données de sources secondaires constitue la première étape de cette recherche. 
Les documents ont été consultés dans différentes bibliothèques à Yaoundé et à Bangangté. Cette phase exploratoire a permis de confronter les problématiques abordées sur cette thématique et de disposer des données sur l'état des lieux de l'approvisionnement en eau dans la Commune de Bangangté. En plus des bibliothèques, l'Internet a permis d'exploiter les articles et les ouvrages ayant trait au sujet. Cette phase a été suivie de la collecte des données de sources primaires dans la Commune de Bangangté qui couvre une superficie d'environ $800 \mathrm{~km}^{2}$ (figure 1). 


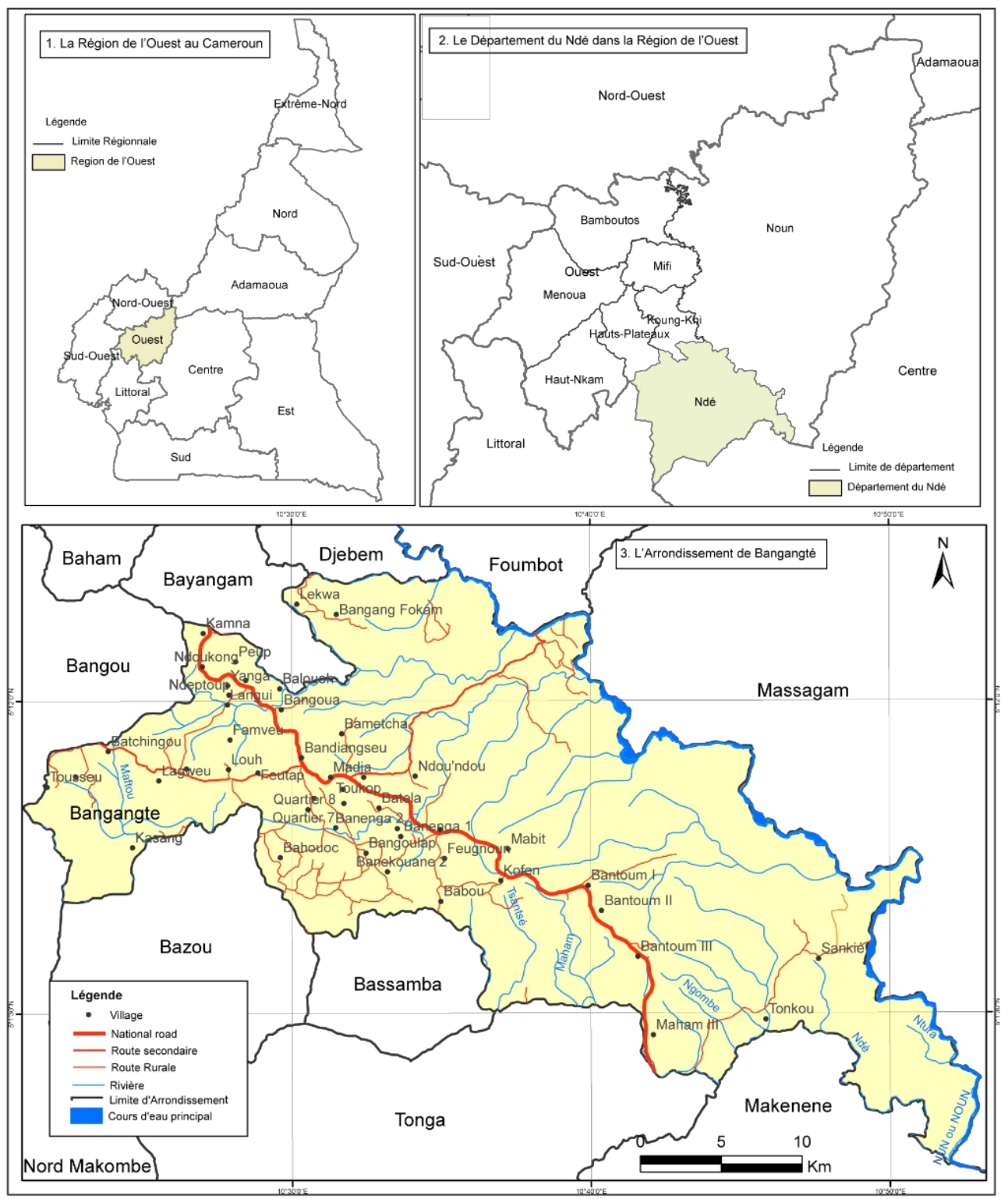

Source : Base de données INS 2014

Figure 1 : Localisation de la zone d'étude

\section{I.1.2. Travaux de terrain}

La collecte des données de sources primaires a consisté à faire des observations de terrain, effectuer des entretiens avec des personnes ressources, conduire des enquêtes auprès des chefs de ménage, faire des inspections sanitaires, prélever des échantillons d'eau pour les analyses et consulter les registres de santé. 


\section{Observations directes}

C'est une technique de recueil des données basée sur la perception visuelle (Quivy et al., 1995). Elle s'est faite à chaque descente sur le terrain. Les observations directes ont été l'occasion de noter les pratiques d'approvisionnement en eau dans la Commune de Bangangté, de réaliser des prises de vues pour illustrer certains faits saillants et d'identifier les personnes ressources

\section{Entretiens}

À l'aide d'un guide d'interview, des entretiens ont été conduits auprès des responsables de la régie communale de l'eau de Bangangté, du service de la production industrielle du réseau Camwater et des Comités des Usagers de l'Eau (CUE) pour cerner les problèmes quotidiens d'approvisionnement en eau et disposer des données quantitatives sur le fonctionnement des réseaux Camwater et Scanwater.

\section{Enquête-ménage}

Les ménages constituent la population cible de cette étude. La Commune de Bangangté compte 7 villages (Bangangté, Bamena, Bangoua, Batchingou, Bangoulap, Bahouoc, Bangang-Fokam). Dans le souci de mener une étude comparative, un tirage à 3 degrés a été effectué. Au premier degré, 4 villages (Bangangté, Bamena, Bangoua et Batchingou) ont été tirés. Au deuxième degré, 10 quartiers ont été sélectionnés, soit 4 en zone urbaine et 6 en zone rurale. En dernier ressort, en se basant sur les résultats du troisième recensement général de la population, un échantillon aléatoire simple (Gumuchian et al., 2000) a été constitué. De ce fait, le questionnaire a été administré à 339 chefs de ménage dont 231 dans la ville de Bangangté, 50 à Bamena, 38 à Bangoua et 20 à Batchingou.

\section{Inspections sanitaires}

Les inspections sanitaires sont une activité exploratrice sur le terrain, de toutes les installations composant un système d'approvisionnement en eau potable, ainsi que les pratiques touchant ce système qui pourraient être à l'origine des risques sur la santé et le bien-être du consommateur (OMS, 2007). Elles ont été réalisées pour décrire l'environnement des points d'eau de boisson afin de faire une meilleure interprétation des résultats des analyses physico-chimiques et bactériologiques.

\section{Identification et prélèvement des échantillons d'eau}

Les points d'eau identifiés pour le prélèvement ont été sélectionnés à la suite des enquêtes-ménages, sur la base de la fréquence de leur utilisation et des plaintes des ménages sur la qualité de l'eau. Au total, des prélèvements 
ont été réalisés dans 12 points d'eau en septembre 2016, soit 5 dans la ville de Bangangté, 1 à Bangoua, 3 à Bamena et 3 à Batchingou (tableau 1).

Tableau 1 : Site de prélèvement des eaux de consommation dans la Commune de Bangangté

\begin{tabular}{|c|c|c|c|c|}
\hline $\begin{array}{l}\text { Cod } \\
\text { e }\end{array}$ & Villages & Quartiers & Dénomination & Observations \\
\hline P1 & \multirow{5}{*}{ Bangangté } & \multirow[t]{2}{*}{ Batéla } & Eau brute Ngam $(\mathrm{C} 1)$ & $\begin{array}{l}\text { Cours d'eau captée par la CW et utilisée } \\
\text { pour la boisson par les populations de } \\
\text { Batéla }\end{array}$ \\
\hline $\mathrm{P} 2$ & & & Eau station Camwater (R1) & Eau traitée au niveau de la station \\
\hline P3 & & Noumfam & Eau robinet Camwater $(\mathrm{R} 2)$ & $\begin{array}{l}\text { Couleur rougeâtre de l'eau au robinet du } \\
\text { consommateur }\end{array}$ \\
\hline P4 & & \multirow[t]{2}{*}{ Mba } & Source non aménagée (S1) & $\begin{array}{l}\text { Source non aménagée située près d'un } \\
\text { ruisseau où les populations font la } \\
\text { lessive et l'utilisent comme boisson }\end{array}$ \\
\hline P5 & & & Source aménagée (S2) & $\begin{array}{l}\text { Source aménagée dont la rigole draine } \\
\text { les eaux sales }\end{array}$ \\
\hline P6 & Bangoua & Ndoukong & Source aménagée (S3) & $\begin{array}{l}\text { Source aménagée située loin des } \\
\text { constructions et qui approvisionne les } \\
\text { populations en eau potable }\end{array}$ \\
\hline P7 & \multirow[b]{3}{*}{ Bamena } & \multirow[b]{3}{*}{ Langweu } & Eau station Scanwater (R3) & Eau traitée au niveau de la station SW \\
\hline P8 & & & Eau robinet Scanwater (R4) & Robinet dont l'eau provient d'un forage \\
\hline P9 & & & Source non aménagée (S4) & $\begin{array}{l}\text { Source non aménagée située dans un lieu } \\
\text { sacré et qui approvisionne les } \\
\text { populations en cas de coupures d'eau ou } \\
\text { les populations ne bénéficiant pas d'un } \\
\text { branchement individuel }\end{array}$ \\
\hline P10 & \multirow{3}{*}{ Batchingou } & \multirow[b]{2}{*}{ Tousseu } & Eau robinet Scanwater (R5) & $\begin{array}{l}\text { Robinet dont l'eau traitée provient d'un } \\
\text { captage gravitaire sur le mont et réalisé } \\
\text { par la Commune de Bangangté }\end{array}$ \\
\hline P11 & & & Borne-fontaine Tousseu (BF1) & $\begin{array}{l}\text { Borne-fontaine dont l'eau provient du } \\
\text { captage villageois non entretenu et est } \\
\text { consommée par les populations du } \\
\text { quartier }\end{array}$ \\
\hline $\mathrm{P} 12$ & & Tougweu & Borne-fontaine Doumbock (BF2) & $\begin{array}{l}\text { Borne-fontaine dont l'eau provient du } \\
\text { captage à la source Doumbock (Mont } \\
\text { Batchingou) }\end{array}$ \\
\hline
\end{tabular}

Source : Investigations de terrain, 2016

\section{Conditionnement et transport des échantillons d'eau}

Les échantillons d'eau ont été conditionnés dans des bouteilles d'eau minérale de 0,5 litre. Deux bouteilles d'eau glacée de 1,5 litre chacune ont été mises dans une glacière pour maintenir la température de l'eau à $4^{\circ} \mathrm{C}$ comme le recommande l'OMS (1986). Les eaux prélevées ont été ensuite convoyées aux laboratoires d'analyse e.

\section{Analyse Physico-chimique}

Les paramètres majeurs qui permettent de valider la qualité physique et chimique de l'eau de boisson ont été choisis. Il s'agit notamment : du potentiel d'hydrogène $(\mathrm{pH})$, des matières en suspension (MES) et de la turbidité. L'analyse a été effectuée au laboratoire des sols et chimie de 
l'environnement de la Faculté d'Agronomie et des Sciences Agricoles (FASA) de l'Université de Dschang. Les résultats de cette analyse ont permis de s'appesantir sur les méthodes de potabilisation à proposer aux ménages de la commune. L'analyse physico-chimique a été complétée par l'analyse microbiologique.

\section{Analyse bactériologique}

L'analyse des paramètres bactériologiques a permis d'identifier les sources de contamination de l'eau et les facteurs explicatifs de l'incidence des maladies d'origine hydrique. Cette analyse a porté sur 4 paramètres: Escherichia coli (E. coli), Streptocoques fécaux (SF), Salmonelles et Shigelles. L'analyse des échantillons d'eau a été faite par le laboratoire de Physiologie animale et microbiologie du Département de la Production Animale de la faculté suscitée. Le dénombrement des germes suivant le procédé de nombre le plus probable (NPP) est la méthode utilisée pour détecter le nombre de colonies dans $100 \mathrm{ml}$ d'eau.

\section{Données sanitaires}

Les informations sur les maladies liées à la consommation d'une eau de mauvaise qualité recueillies dans les ménages ont été complétées par celles collectées dans les centres de santé intégrés (CSI) des 4 villages.

\section{I.2. Traitement et analyse des données}

Les données issues des sources secondaires et des entretiens ont été traitées suivant la méthode de l'analyse de contenu (Quivy et al., 1995). Les données obtenues à base du questionnaire ont été codifiées et enregistrées dans un masque de saisie réalisée avec l'application KoboCollect. Par la suite, les données ont été exportées pour traitement dans le logiciel SPSS version 20. Ainsi, des tableaux ont été générés. Excel 2007 a permis la réalisation des figures. Le logiciel ArcGis 10.2.2 a été mis à contribution pour le traitement cartographique.

\section{Résultats et discussions}

\section{II.1. Un réseau dysfonctionnel de l'approvisionnement en eau potable dans la ville de Bangangté}

De la création du réseau Camwater en 1978 dans la ville de Bangangté jusqu'à nos jours, la production n'a jamais pu satisfaire les besoins exprimés par la population. Au fil des années, la situation s'est aggravée avec le vieillissement des équipements et les coupures régulières de l'énergie électrique qui impactent grandement les activités de production et de distribution (tableau 2). 
Tableau 2 : Évolution des performances du réseau Camwater dans la ville deBangangté

\begin{tabular}{|c|c|c|c|c|c|}
\hline Années & $\begin{array}{l}\text { Production } \\
\left(\mathbf{m}^{\mathbf{3}}\right)\end{array}$ & $\begin{array}{l}\text { Distribution. } \\
\left(\mathbf{m}^{\mathbf{3}}\right)\end{array}$ & Perte $\left(\mathrm{m}^{3}\right)$ & $\begin{array}{lr}\begin{array}{l}\text { Déficit } \\
\text { production }\end{array} & \text { de } \\
\text { imputable } & \text { aux } \\
\text { coupures }\left(\mathrm{m}^{3}\right) & \end{array}$ & $\begin{array}{l}\text { Déficit de production } \\
\text { Imputable aux pannes } \\
\text { électromagnétiques }\end{array}$ \\
\hline 2012 & 246996 & 177783 & 69213 & 52205 & 23298 \\
\hline 2013 & 210747 & 158955 & 51792 & 62718 & 15810 \\
\hline 2014 & 338442 & 195171 & 143271 & 82532 & 8638 \\
\hline 2015 & 261487 & 191704 & 69783 & 111344 & 18255 \\
\hline 2016 & 313893 & 219837 & 94056 & 62863 & 14147 \\
\hline 2017 & 523878 & 294404 & 229474 & 114401 & 2682 \\
\hline 2018 & 415748 & 230206 & 185542 & 56055 & 1320 \\
\hline
\end{tabular}

Source : Reporting CDE (2019), investigations de terrain (2016-2019)

Il ressort de l'analyse de ce tableau que la production de 2012 à septembre 2018 a nettement évolué grâce au Projet d'Alimentation en Eau Potable et Assainissement en Milieu Semi-Urbain (PAEPA-MSU) réalisé en 2016. Ainsi, elle est passée de $261487 \mathrm{~m}^{3}$ en 2015 à $415748 \mathrm{~m}^{3}$ en septembre 2018. Cette production aurait été plus élevée n'eurent été les pannes électromagnétiques et le délestage. Avec la construction de nouveaux équipements, les pannes sont de moins en moins fréquentes. Le déficit de production est passé de $18255 \mathrm{~m}^{3}$ en 2015 à $1320 \mathrm{~m}^{3}$ en 2018. Cependant, les coupures de l'énergie électrique s'accentuent. En 2017, elles ont empêché de produire $114401 \mathrm{~m}^{3}$ d'eau. La production demeure insuffisante pour satisfaire les besoins d'une population estimée à 100000 habitants. À la production insuffisante se greffent les pertes enregistrées dans la distribution.

Pour maîtriser les fuites, et mieux gérer les faibles quantités d'eau produite, la Camwater est obligée de procéder à un rationnement et priver aussi certains quartiers de Bangangté (Mba et Sagnam) de ce précieux liquide. Dans ce contexte, les abonnés ne peuvent pas bénéficier du service en permanence (figure 2). L'eau arrive 2 à 3 fois par semaine. 


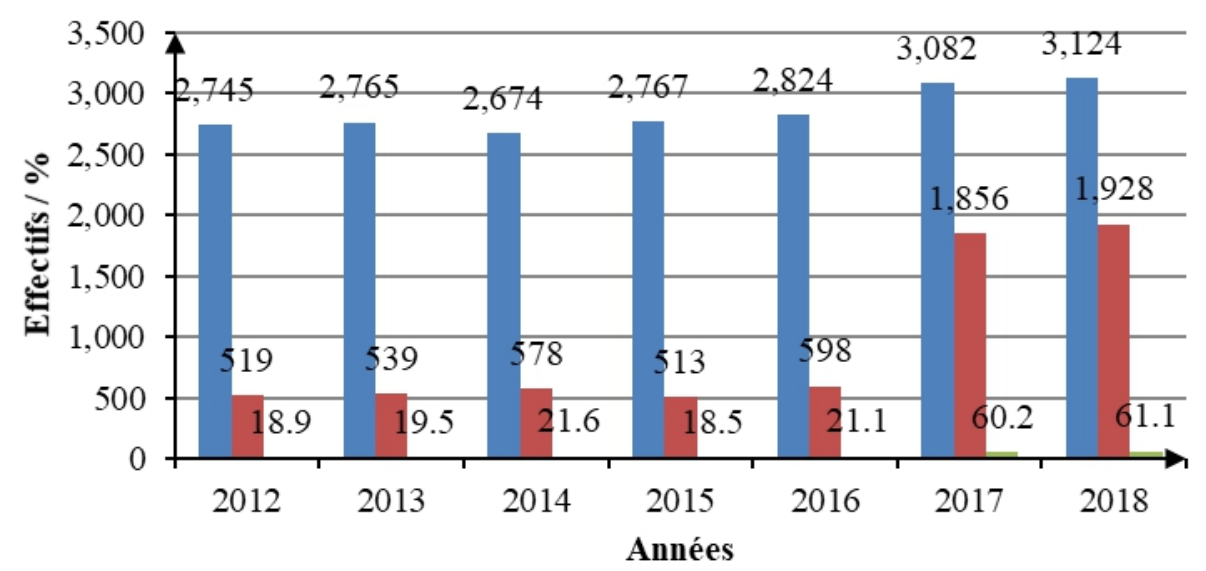

- Abonnés actifs

- Aboonés actifs desservis $24 / 24$

n $\%$ des aboonés actifs desservis

Source : Reporting CDE (2019), investigations de terrain (2016-2019)

Figure 2 : Évolution de la clientèle et niveau de service d'approvisionnement en eau

Les populations dans la ville de Bangangté, ne sont pas motivées à solliciter un branchement au réseau. Cette démotivation n'est aucunement liée à la cherté du branchement, mais à la difficulté du concessionnaire à satisfaire les attentes. Malgré l'évolution remarquable observée dans la disponibilité du service, $38,9 \%$ des abonnés ne disposent pas de l'eau au moins 12 heures par jours.

Pour pallier l'indisponibilité du service et la qualité de l'eau douteuse, les populations sollicitent l'eau des sources pour la boisson, et les plus nanties, les forages tandis que les eaux des puits sont utilisées pour les usages domestiques.

\section{II.2. Une fourniture d'eau potable par Scanwater adaptée aux populations à revenus moyens}

En zone rurale, les forages Scanwater, qui autrefois alimentaient les populations, ont cessé de fonctionner dans les années 2000, faute de maintenance et d'entretien. Grâce à la coopération internationale, le réseau Scanwater dans les villages de la Commune de Bangangté a été remis en service à partir de 2006. Dans la localité de Batchingou, le choix a porté sur le captage d'eau gravitaire. Les populations sont approvisionnées par des branchements individuels. À Sanki, par contre, la pompe électrique a été remplacée par une pompe solaire régulièrement en panne, perturbant ainsi la production de l'eau potable pendant des mois. Les populations s'approvisionnent à partir de 2 bornes-fontaines. À Bantoum 3, les ménages 
s'approvisionnent à une borne-fontaine dont l'eau provient d'un puits motorisé construit par la commune et le PNDP. Dans le village Bangoua, le réseau Scanwater n'est plus fonctionnel à cause de la mésentente entre la Commune de Bangangté et le chef du village sur le mode de gestion. De ce fait, chaque ménage doit se débrouiller pour son approvisionnement.

Dans les villages Bamena et Batchingou, le réseau, bien que fonctionnel, compte peu d'abonnés actifs (figure 3).

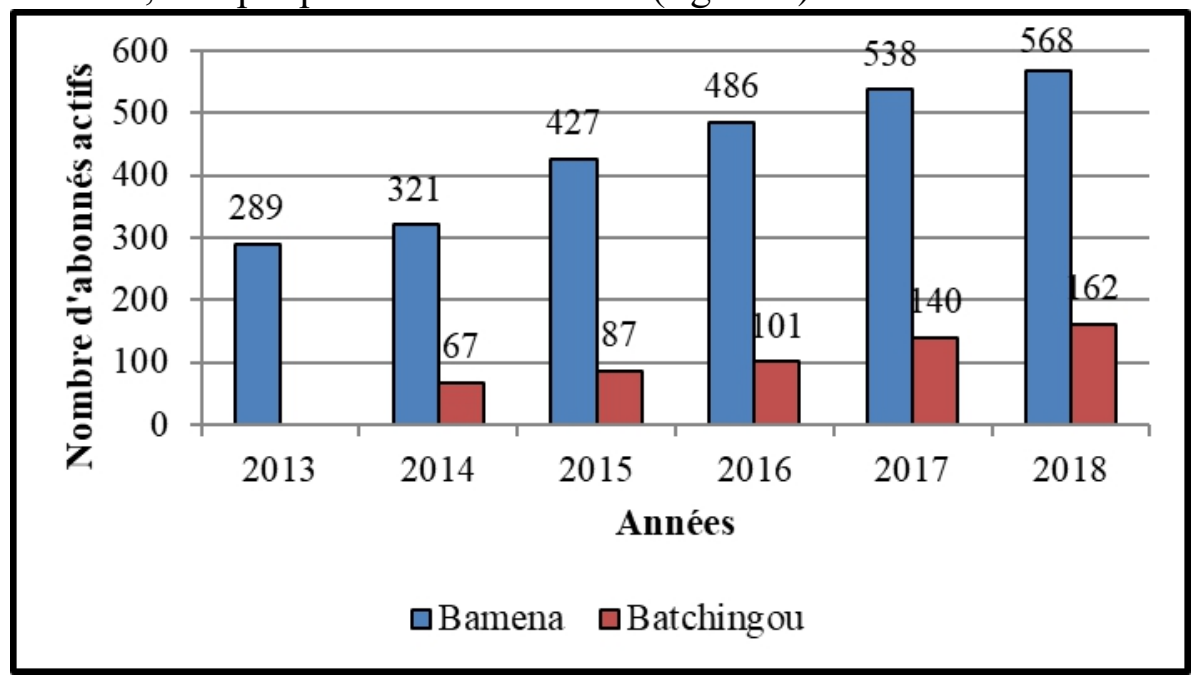

Source : Régie communale de l'eau, investigations de terrain (2016-2019)

Figure 3 : Évolution du nombre des abonnés actifs à Bamena et Batchingou

Il se dégage de la figure 3 que le rythme d'évolution des abonnés actifs à Batchingou est lent par rapport à celui de Bamena. La coexistence de deux réseaux parallèles d'approvisionnement gravitaire en est la cause. Les populations de Bangou-carrefour et une partie de Bamena, sont les principaux abonnés à ce réseau. Le coût du branchement varie en fonction de la proximité du ménage à la canalisation principale. Pour bénéficier du service public de distribution de l'eau potable dans les deux villages, il faut verser une somme de 60000 F CFA répartie comme suit : 10000 F CFA sont les frais d'abonnement et 50 000F CFA les frais de branchement, y compris le matériel d'installation pour $10 \mathrm{ml}$. À plus de $10 \mathrm{ml}$, le coût du branchement devient élevé. Le $\mathrm{m}^{3}$ de l'eau est facturé à $350 \mathrm{~F} \mathrm{CFA} \mathrm{dans} \mathrm{les} 2$ villages. Par contre, l'entretien compteur à Bamena s'élève à $1000 \mathrm{~F}$ et $500 \mathrm{~F} \mathrm{CFA}$ à Batchingou. Pour bénéficier de ce service, les abonnés doivent mobiliser d'importantes sommes d'argent.

Dans ces 2 villages, le prix du branchement, du $\mathrm{m}^{3}$ d'eau et de l'entretien compteur est élevé pour une population pauvre pratiquant majoritairement une agriculture de subsistance. Limitées par les moyens financiers, suivis des mécontentements liés au système de gestion mis en place 
par la commune, les populations rurales se tournent vers les réseaux où le service est gratuit et où l'eau est disponible à tout moment.

\section{II.3. Des solutions autonomes d'approvisionnement en eau dans la Commune de Bangangté}

Dans l'ensemble, les eaux de sources, forages, et bornes-fontaines sont sollicitées pour la boisson et celles de puits, rivières et pluies pour les activités domestiques. Les sources, qu'elles soient aménagées ou non sont nombreuses (environ 15). En saison sèche, leur débit baisse tandis que la demande en eau est croissante. Avec les coupures fréquentes des réseaux Camwater et Scanwater, ces points d'eau sont saturés (photos 1). Cependant, les sources ne bénéficient pas d'un périmètre de protection et sont par conséquent exposées à des pollutions d'origines diverses.

Les puits sont plus répandus dans la ville de Banagangté (photo 2). Sur 231 ménages interrogés, 39 disposent d'un puits aménagé, 25 un puits sommairement aménagé et 4 un puits non aménagé (figure 4). Les ménages adoptent aussi ce mode d'alimentation pour pallier les coupures de longue durée du concessionnaire.
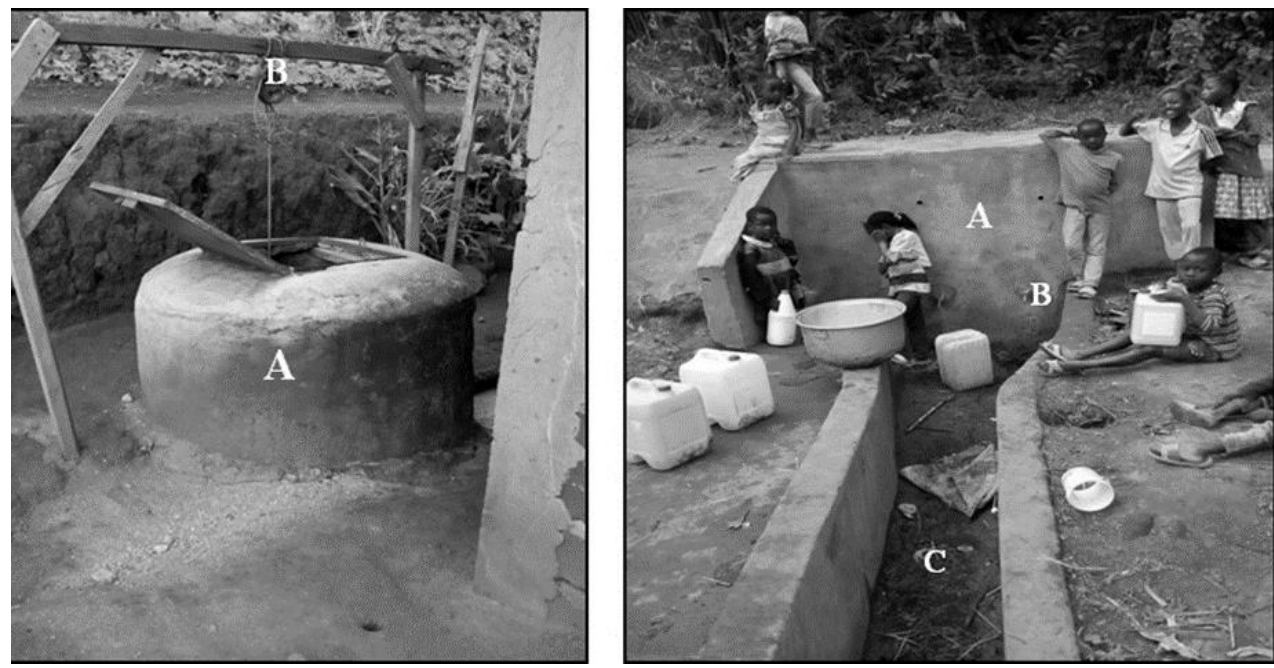

Planche 1 : Points d'eau les plus sollicités à Bangangté

Septembre 2016

Photo 1 : Puits aménagé au quartier 2 $\boldsymbol{A}:$ Margelle qui assure une protection moyenne de l'ouvrage. $\boldsymbol{B}$ : Système de poulie mis en place pour la remontée de l'eau.
Août 2016 Photo 2 : Source aménagée à Bangoua $\boldsymbol{A}$ : Bac en béton de retenue d'eau captée. $\boldsymbol{B}$ : Robinet rendant l'écoulement de l'eau possible. C: Rigole couverte de dalle bétonnée pour le drainage des eaux usées .

Les forages équipés d'une pompe à motricité humaine réalisés dans le cadre des Budget d'Investissement Public ou sur fonds propres de la commune 
sont pour la plupart en pannes. Ceux qui fonctionnent sont en majorité réalisés par des particuliers qui installent des bornes-fontaines pour vendre l'eau, soit la distribuer gratuitement aux populations. En zone rurale comme en zone urbaine, les populations pour économiser l'eau et réduire la corvée, font généralement la lessive, la vaisselle et parfois la douche à la rivière ou à la source de captage.

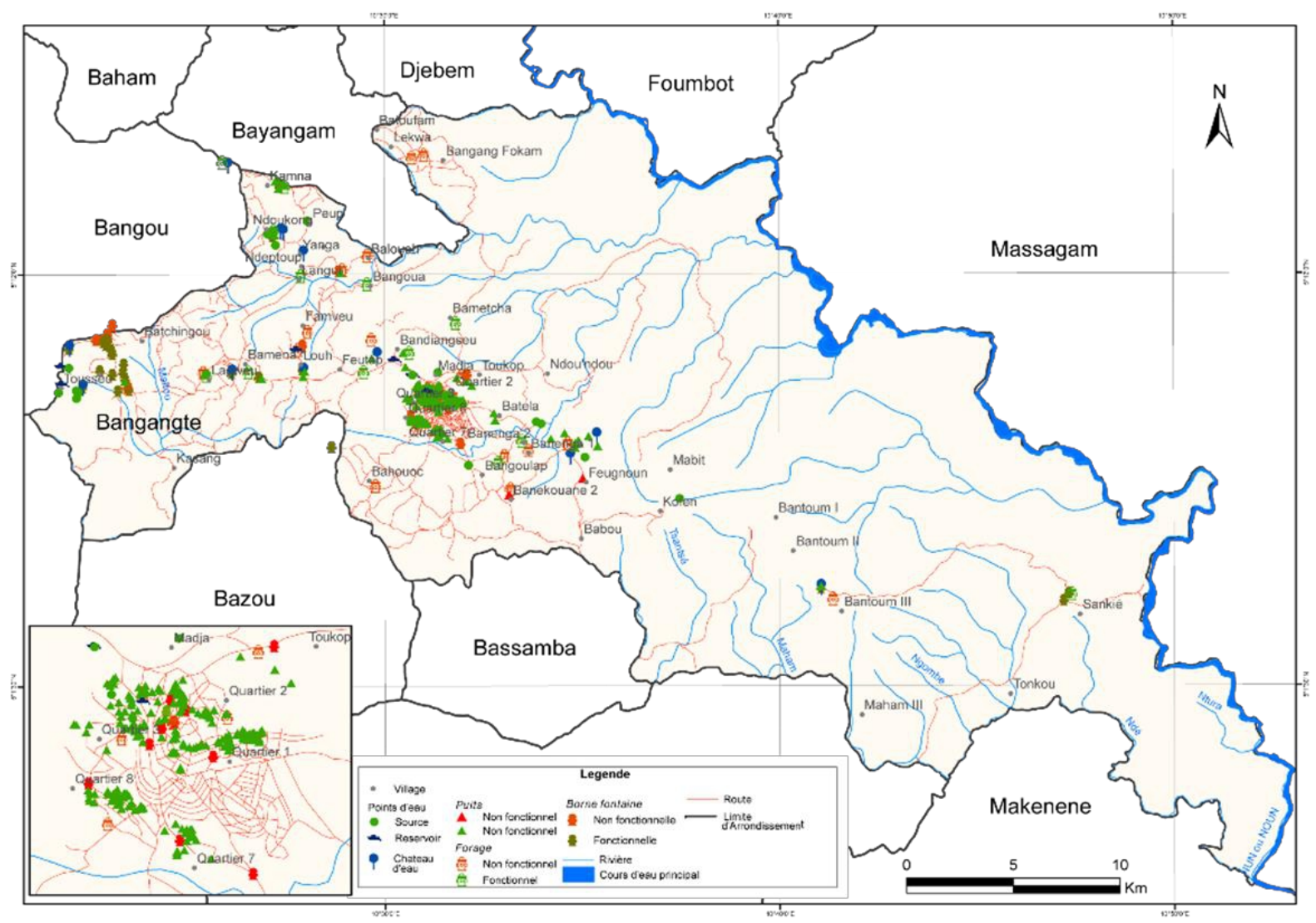

Source : Investigations de terrain, 2016-2018

Figure 4 : Répartition spatiale des modes d'approvisionnement en eau dans la Commune de Bangangté

Pour résoudre les problèmes liés à leur approvisionnement en eau, les populations choisissent des solutions individuelles en fonction de leurs revenus, de la disponibilité du réseau, de l'accessibilité physique aux points d'eau et de la distance à parcourir pour s'approvisionner. L'option adoptée pour se ravitailler en eau les rend vulnérables à la contraction des maladies d'origine hydrique. 


\section{II.4. Qualité de l'eau consommée par les populations et répercussions sur la santé}

\section{II.4.1. Qualité des eaux de consommation}

Les paramètres physico-bactériologiques varient en fonction de la nature des points d'eau prélevés et du traitement administré. Dans l'eau du réseau Camwater, les matières en suspension qui selon l'OMS (1985) doivent être absentes dans une eau destinée à la consommation humaine sont présentes après traitement $(2,1 \mathrm{mg} / \mathrm{l})$. Elles sont absentes dans les eaux de sources et robinets, à l'exception de la borne-fontaine du captage villageois à Batchingou où leur concentration est très élevée $(13,2 \mathrm{mg} / \mathrm{l})$. L'eau traitée à la station de captage de Batéla, lors de la distribution, a une turbidité de 10,7 NTU (figure $5)$.

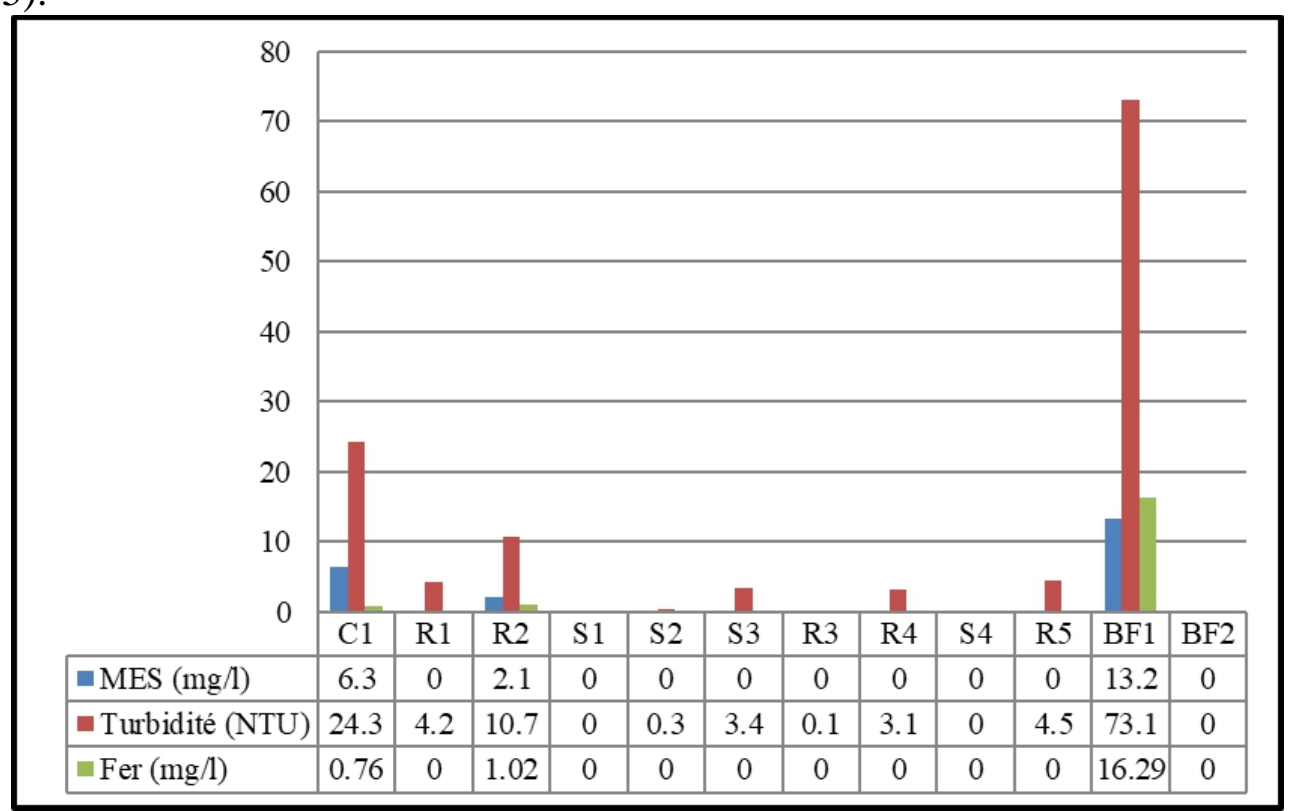

Source : Investigations de terrain, 2016

Figure 5 : Caractéristiques physico-chimiques des eaux de consommation

La turbidité la plus élevée est enregistrée dans l'eau provenant du captage villageois $(73,1 \mathrm{NTU})$. Une turbidité supérieure à 5 NTU pourrait, d'une part, empêcher la détection des bactéries et des virus en les absorbant dans les particules et d'autre part, protéger les microorganismes contre l'action des désinfectants. L'OMS recommande une valeur limite de 5 NTU et précise que la turbidité doit être $<1$ pour une désinfection efficace (WHO, 1997). Le fer est présent dans 2 échantillons d'eau ayant reçu un traitement. Sa concentration est de $1,02 \mathrm{mg} / \mathrm{l}$ dans l'eau du robinet Camwater et $16,29 \mathrm{mg} / \mathrm{l}$ à la borne-fontaine du captage villageois. Pour des raisons de commodités, 
l'OMS recommande une valeur guide de $0,3 \mathrm{mg} / \mathrm{l}$. Dans la ville de Bangangté, la population évite, lorsque la couleur n'est pas bonne, d'utiliser l'eau du réseau Camwater pour faire la lessive. Dans l'ensemble, les paramètres physiques de l'eau Camwater après traitement et celle du captage villageois non traitée ne sont pas conformes aux normes de l'OMS.

Les résultats de l'analyse bactériologique montrent que l'eau consommée par les populations contient des bactéries responsables de la transmission des maladies hydriques (figure 6).

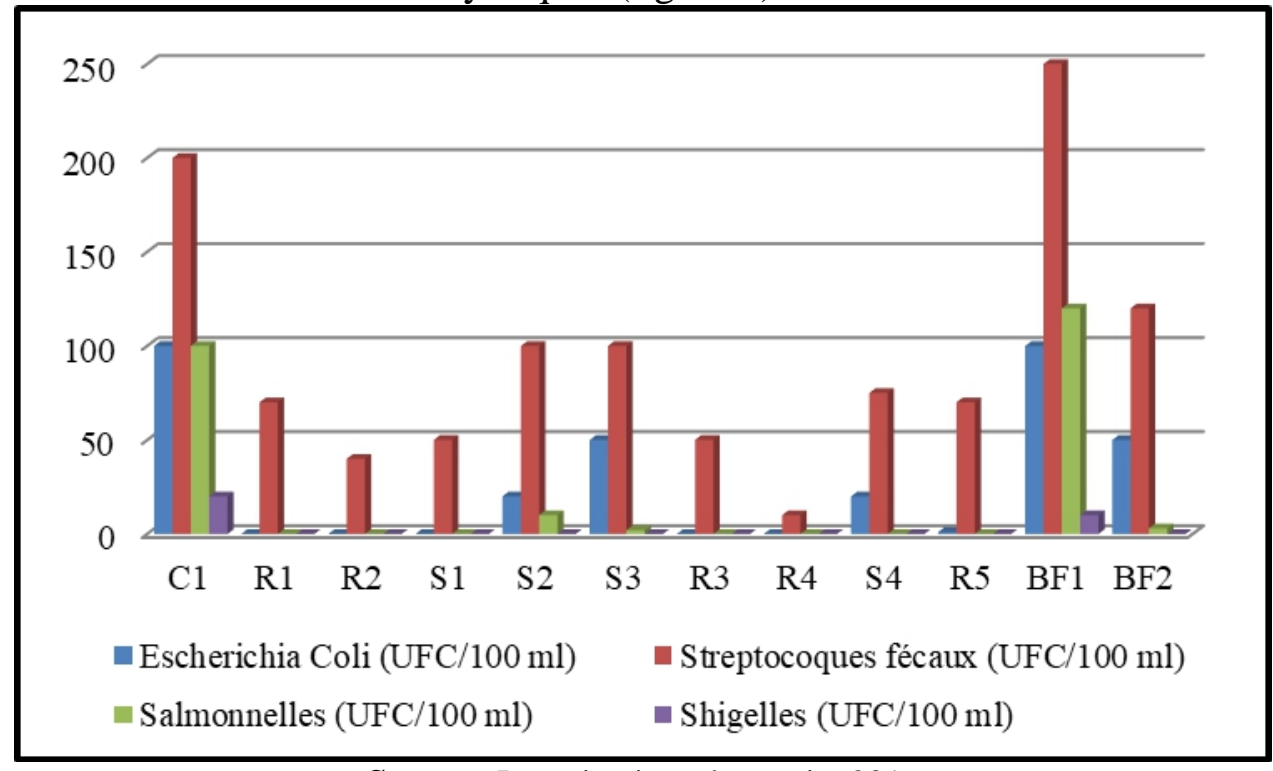

Source : Investigations de terrain, 2016

Figure 6 : Caractéristiques bactériologiques des eaux de consommation

La présence d'Escherichia coli dans une eau destinée à la boisson est la preuve d'une contamination par les fèces. Cette bactérie est l'agent responsable de la diarrhée. Elle est absente après traitement de l'eau Camwater et présente à faible concentration dans l'eau de la borne-fontaine Scanwater à Batchingou. Sa concentration est élevée dans les eaux de sources où elle varie de $20 \mathrm{UFC} / 100 \mathrm{ml}$ à la source aménagée de Bangangté à $100 \mathrm{UFC} / 100 \mathrm{ml}$ à la borne-fontaine du captage villageois. Que l'eau soit traitée ou non, la concentration en streptocoque est élevée dans tous les échantillons. La concentration minimale est enregistrée à la borne-fontaine Scanwater de Bamena (10 UFC/100 ml) et maximale à la borne-fontaine du captage villageois $(250 \mathrm{UFC} / 100 \mathrm{ml})$. Les salmonelles sont présentes seulement dans 5 échantillons. Elles sont à l'origine de la fièvre typhoïde. Leur concentration varie de 2 UFC/100 ml à la source aménagée à Bangoua à 120 UFC/100 ml à la borne-fontaine du captage villageois. Les shigelles sont responsables de la 
dysenterie bacillaire. Elles sont présentes dans 2 échantillons d'eau, mais à des concentrations peu élevées.

Il ressort des résultats de l'analyse de l'eau à Bangangté, que les points d'eau sollicités par les populations pour pallier les difficultés liées à leur approvisionnement, bien que satisfaisants au regard de leur perception, les exposent à la contraction des maladies hydriques. La conséquence de la consommation de ces eaux est la récurrence des maladies hydriques.

\section{II.4.2. Maladies hydriques fréquentes dans la Commune de Bangangté}

Dans les localités où les enquêtes ont été effectuées, la typhoïde, les diarrhées et l'amibiase sont les trois maladies hydriques qui affectent les ménages de façon récurrente (figure 7).

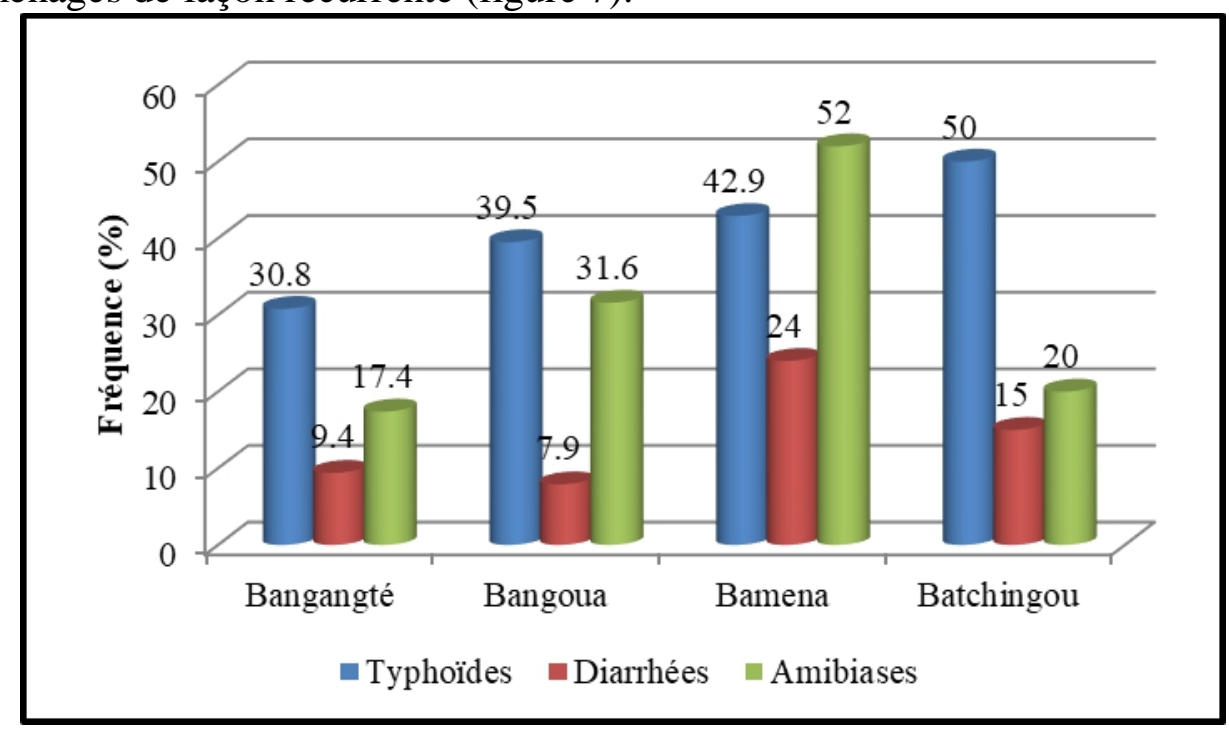

Source : Investigations de terrain, 2016

Figure 7 : Répartition des maladies hydriques dans la Commune de Bangangté

La typhoïde est très répandue. Elle affecte plus les ménages de Bamena et de Batchingou soit respectivement $42,9 \%$ et $50 \%$. L'amibiase sévit plus à Bamena (52\%) et à Bangoua $(31,6 \%)$.

Ces données ont été complétées pas celles relevées dans les registres de santé des différentes localités. Il ressort du tableau 3 que le nombre de patients souffrant de la typhoïde (344 cas) est élevé dans les 4 formations sanitaires. Les cas des malades de l'amibiase sont peu diagnostiqués parce que la plupart des patients se soignent avec des potions traditionnelles et ne se rendent dans les hôpitaux que lorsque la maladie persiste. Les patients qui souffrent de la diarrhée sont plus enregistrés au CSI de Bangangté. L'utilisation des eaux de rivière pour la lessive, la vaisselle et la baignade est à l'origine des helminthiases (133 cas) et des problèmes de peau (50 cas) qui 
affectent les ménages. L'utilisation de ces eaux est la conséquence de l'indisponibilité et de l'inaccessibilité des réseaux publics de distribution d'eau potable.

Tableau 3 : Maladies hydriques recensées en 2015 dans les centres de santé intégrés

\begin{tabular}{|l|l|l|l|l|}
\hline Pathologies & CSI de Bangangté & CSI de Bangoua & CSI de Bamena & CSI de Batchingou \\
\hline Typhoïdes & 151 & 129 & 47 & 17 \\
\hline Amibiases & 9 & 3 & 4 & 10 \\
\hline Diarrhées & 12 & 8 & 5 & 1 \\
\hline Helminthiases & 39 & 45 & 39 & 10 \\
\hline Dermatoses & 11 & 10 & 23 & 6 \\
\hline
\end{tabular}

Source : CSI des 4 localités (2018)

La relation entre l'eau consommée et la récurrence des maladies hydriques est évidente. L'enquête-ménage révèle que les eaux de sources et de bornes-fontaines sont les plus consommées (figure 8).

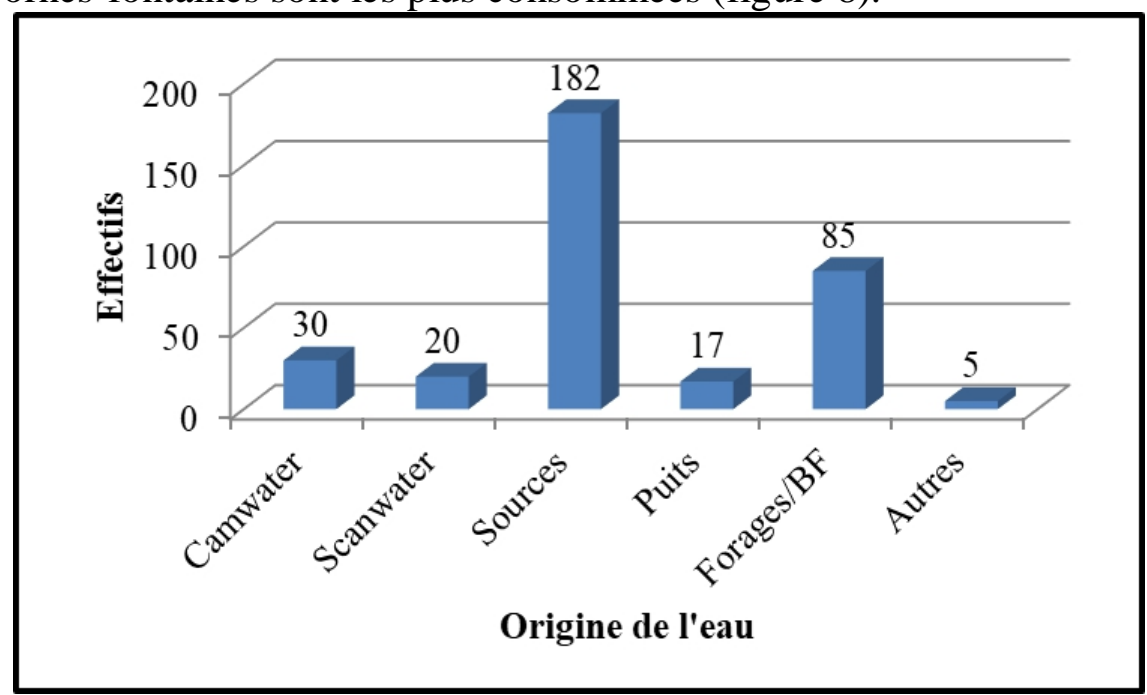

Source : Investigations de terrain, 2016

Figure 8 : Principal mode d'approvisionnement en eau de boisson

Il ressort de l'analyse de la figure 8 que sur 339 ménages interrogés, 182 s'approvisionnent pour la boisson aux sources et 85 plus aux bornesfontaines qu'aux forages. Or, les résultats de l'analyse ont montré que les eaux provenant de ces ouvrages contiennent des bactéries nuisibles à la santé humaine.

\section{Discussions}

Dans la Commune de Bangangté, les populations urbaines s'approvisionnent majoritairement aux puits et aux sources à cause de la faible production de l'eau potable, de la discontinuité du service et de la faible extension du réseau Camwater. La production insuffisante est liée à la baisse 
progressive du débit du cours d'eau Ngam dont les eaux sont captées et aux coupures fréquentes de l'énergie électrique. En effet, les travaux de Tchindjang (1985) et Tchawa et al (2002) révèlent que la végétation d'Eucalyptus est à l'origine de la baisse du débit des sources d'eau sur les hautes terres de l'Ouest. Le délestage n'est pas un problème local. Guéye (2012) montre que dans les pays en développement, la fourniture déficitaire de l'énergie électrique devient de plus en plus une contrainte majeure pour la production, le traitement et la distribution de l'eau potable. Le relief accidenté de la commune ne favorise pas l'extension du réseau Camwater. De ce fait, les quartiers Mba et Sagnam à Bangangté situés à une altitude élevée par rapport au réservoir de distribution sont privés d'eau potable.

Dans la zone rurale, le coût constitue une contrainte majeure à l'accès à l'eau potable. Pourtant, la cible 6.1 des Objectifs du Développement Durable (ODD) stipule que le payement des services ne doit pas représenter un obstacle à l'accès à l'eau ou empêcher les individus de répondre à d'autres besoins fondamentaux (Genevaux, 2018). Le recours aux eaux provenant des points d'eau non améliorés à Bangangté est un phénomène observé dans de nombreuses villes africaines.

Cette solution envisagée par les populations constitue un risque pour leur santé. La turbidité élevée de l'eau Camwater après traitement est la preuve de l'inefficacité de la désinfection, de la faible pression de l'eau dans le réseau d'alimentation, de l'exploitation intermittente et des fuites au niveau des canalisations qui causent l'infiltration d'agents contaminants (OMS, 1986). La présence du fer dans l'eau à un certain seuil, peut induire des désagréments (couleur, goût) qui peuvent entraîner le détournement de l'usager vers une eau de mauvaise qualité (Viland et al. 2001). La forte concentration du fer dans le réseau de distribution surtout de Bangangté et la borne-fontaine du captage villageois de Batchingou provient de la corrosion des conduites d'amenées. Par ailleurs, les sources de captage ne sont pas sécurisées par une barrière pouvant empêcher le passage des animaux en divagation.

Les résultats sur la qualité de l'eau viennent confirmer le constat des différents usagers de cette ressource dans la Commune de Bangangté. En effet, $21,8 \%$ des ménages interrogés pensent que l'eau qu'ils consomment est de mauvaise qualité. Les résultats de l'analyse bactériologique corroborent ceux obtenus en 2012 par Kemayou, sur les eaux des sources à Bangangté, Nana et Tchoumkeu (2014) sur la qualité des eaux du réseau Scanwater des villages Bamena et Batchingou et Njikeu (2015) sur la qualité des eaux de surface et souterraines dans la ville de Tonga. Ces auteurs étaient parvenus à la conclusion selon laquelle ces eaux étaient d'une qualité médiocre et qu'un traitement préalable était indiqué avant toute consommation humaine. 


\section{Conclusion}

L'accès de tous à l'eau potable est un droit fondamental que vise l'ODD $\mathrm{n}^{\circ} 6$. Malheureusement, les contraintes d'ordre naturel, technique et financier dans la Commune de Bangangté poussent les populations à s'approvisionner en eau de boisson dans les points d'eau non améliorés. L'analyse des échantillons prélevés montre que les eaux sont colonisées par Escherichia coli, streptocoques fécaux, salmonelles et shigelles. Les eaux de certains réseaux ne présentent pas de bonnes caractéristiques physiques. La typhoïde, les diarrhées, l'amibiase, les helminthiases et les dermatoses sont la conséquence de la consommation de ces eaux de mauvaise qualité. La réduction de ces maladies passe par la construction des ouvrages d'eau et d'assainissement de qualité et l'amélioration de la qualité de l'eau. Si l'on veut donner la possibilité aux populations de cette commune d'atteindre les ODD $n^{\circ} 6$ (eau propre et assainissement) et 3 (bonne santé et bien-être), les interventions doivent privilégier les actions visant la prévention ou la réduction des risques à la source de captage. Sécuriser les sources de captage d'eau les plus fréquentées à travers la délimitation d'un périmètre de protection, analyser régulièrement les eaux destinées à la boisson, sensibiliser et éduquer les populations sur les méthodes de traitement de l'eau à domicile et le respect des pratiques d'hygiène et d'assainissement sont des actions pratiques et nécessaires. Le traitement de l'eau par décantation, filtration et désinfection est un impératif. La désinfection peut se faire avec l'eau de javel, le comprimé aquatabs et le soleil.

\section{References:}

1. Adeline T., Amirault P., (1994). Réhabilitation des points d'eau populaires dans une grande métropole: le cas de Yaoundé. Les éditions du Gret, pp. 126-128

2. Beninguisse G., (1993). Approvisionnement en eau et assainissement : effets sur la santé des enfants par maladies diarrhéiques. Le cas du Cameroun. D.E.D, IFORD, 89 p.

3. Djeuda Tchapnga H.B., Tanawa E., Ngnikam E., (2001). L'eau au Cameroun. Tome I : Approvisionnement en eau potable. PUY, ENSP, $321 \mathrm{p}$.

4. Génevaux C., (2018). Les Objectifs de Développement Durable pour les services d'eau et d'assainissement: Décryptage des cibles et indicateurs. pS-Eau, 54 p.

5. Guéye A., (2012). Précarité et service d'eau potable et d'assainissement: Les quartiers pauvres de Dakar (Sénégal) à l'épreuve des projets communautaires. Thèse de Doctorat, Université Cheikh Anta Diop, 396 p. 
6. Gumuchian H., Marois C., Fevre V., (2000). Initiation à la recherche en géographie: Aménagement, développement territorial, environnement. Ed ECONOMIC, PUM, Montréal, 425 p.

7. GWP-MINEE, (2009c). État des lieux du secteur : Cadre financier, économique et social. Tome 3, $236 \mathrm{p}$.

8. Kemayou Tchamako E., (2012). Influence de la lithologie et de l'activité anthropique sur la qualité des eaux : Cas du bassin versant du Ngam (Bangangté-Cameroun). Mémoire de Master II, UY 1, 84 p.

9. Mougoué B., (1982). Croissance urbaine périphérique : le cas de la zone-Est de Yaoundé. Thèse de Doctorat $3^{\mathrm{e}}$ cycle, Université de Yaoundé, $300 \mathrm{p}$.

10. Njikeu O., (2015). Influence de la lithologie et des activités anthropiques sur la qualité des eaux du bassin versant du Ndé (OuestCameroun). Mémoire de Master II en Science de la Terre, UY 1, 76 p.

11. Nnanga N., Ngene J.P., Tsala D.E., Ngoula C., Lamare N., (2014). «Relation entre pollution des eaux de sources, forages et maladies hydriques enregistrées au Centre Hospitalier Dominicain Saint Martin de Porres (CHDSMP) du quartier Mvog-Betsi à Yaoundé ». Sci ; Dis., Volume $15, \mathrm{n}^{\circ} 3,8 \mathrm{p}$.

12. Nya E. L., (2014). Approvisionnement en eau et impacts sur la santé des populations de la ville de Bangangté et sa périphérie. Mémoire de Master en Géographie, UY 1, 145 p.

13. OMS, (2007). Guide des inspections sanitaires des systèmes d'alimentation en eau de boisson. 203 p.

14. OMS, (1986), Contrôle de la qualité de l'eau de boisson destinée à l'approvisionnement des petites collectivités, Volume 3, Genève, 134 p.

15. OMS, (1986). Directives de qualité pour l'eau de boisson. Volume 2, Critères d'hygiène et documentation à l'appui, Genève, $341 \mathrm{p}$.

16. OMS, (1985). Directives de qualité pour l'eau de boisson. Volume 1, Genève, $129 \mathrm{p}$.

17. OMS, UNICEF, (2000). Rapport sur l'évaluation de la situation mondiale de l'approvisionnement en eau et de l'assainissement, $80 \mathrm{p}$.

18. Quivyl, Campenhoudt, (1995). Manuel de recherche en sciences sociales. Dunot, Paris, $287 \mathrm{p}$.

19. Tchawa P., Tsayem Demaze M., (2002). «Gestion de l'espace et effet écologique de l'eucalyptus en pays Bamiléké (Ouest-Cameroun) : Stratégie paysanne et prise en compte d'un risque perçu ». Les cahiers d'Outre-mer, Revue de géographie de Bordeaux, 15 p.

20. Tchindjang M., (1985). Le rebord du plateau bamiléké autour de Bangangté. Mémoire de Maîtrise en Géographie physique, UY, 125 p. 
21. Tchoumkeu L. P., (2014). Maîtrise des pertes d'eau potable et analyse de la qualité des eaux des réseaux Scan-Water de la Commune de Bangangté : Cas du groupement de Bamena, Mémoire d'Ingénieur de conception en hydraulique et maîtrise des eaux. Université de Maroua, $65 \mathrm{p}$.

22. UNICEF, (2006). Progrès pour les enfants : Un bilan de l'eau et de l'assainissement. $\mathrm{N}^{\circ} 5,36 \mathrm{p}$.

23. UNICEF, OMS, (2017).Une eau gérée en toute sécurité. Rapport thématique, $56 \mathrm{p}$.

24. Viland M., Montiel A., Duchemin J., Larivière M., Zarrabi P., (2001). Eau et santé: Guide pratique pour les intervenants en milieu rural africain. Études et travaux, GRET, $109 \mathrm{p}$.

25. WHO, (1997). Guidelines for drinking-water quality. Second edition, Volume 3, Geneva, 238 p. 